\title{
Synthesis of novel highly substituted pyrimidines bearing furanyl thiazole nucleous
}

\author{
Haresh G. Kathrotiya, Yogesh T. Naliapara* \\ Department of Chemistry, Saurashtra University, Rajkot-360005, Gujarat, India \\ *E-mail address: naliaparachem@yahoo.co.in
}

Keywords: Pyrimidine; Furanyl; Thiazole

\begin{abstract}
A series of novel highly substituted pyrimidines bearing furanyl thiazole nucleous have been synthesized. In which reaction of guanidine nitrate with ketene dithioacetals $\mathbf{3 c}$ produced substituted pyrimidines $\mathbf{4 d}$ which further treated with various aromatic aldehydes to afford title compounds $\mathbf{5}(\mathbf{a}-\mathbf{t})$. The chemical structures of the synthesized compounds were elucidated by ${ }^{1} \mathrm{H}$ NMR, ${ }^{13} \mathrm{C}$ NMR, FT-IR, elemental analysis, and mass spectral data.
\end{abstract}

\section{INTRODUCTION}

At very early period in the history of organic chemistry, pyrimidines ('m-diazine') were known as the breakdown products of uric acid. The first pyrimidine derivative to be isolated was alloxan in 1818 by Brugnatelli, oxidizing uric acid with nitric acid [1]. The name pyrimidine (combination of words pyridine and amidine) was first applied by Pinner. Pyrimidines are the most important six membered heterocyclic containing 2 nitrogen atoms at position 1 and 3. It is isomeric with two other forms of diazene. Nucleic acid hydrolysis produces several pyrimidines (uracil, thymine and cytosine). Of the 2 types of nucleic acid DNA and RNA, cytosine is found present in both DNA and RNA, while uracil present only in RNA and thymine only in DNA [2].

Pyrimidine derivatives are well known for their pharmacological activities. Various drugs containing pyrimidine nucleus were synthesized and used as anticancer agents like 5-Fluorouracil (5-FU), Tegafur and Thioguanine [3]. An interest in pyrimidine derivatives as anticancer agents has led to the preparation and anticancer activity evaluation of hundreds of such molecules. For example, 2- cyanopyrimidines [4], hydrazino pyrimidine-5- carbonitriles [5], 1,3-dialkylatedpyrimidin-2,4-diones [6] and 4-anilino-2-(2-pyridyl). Pyrimidines were evaluated as a new class of potent anticancer agents [7]. Also, pyrimidine derivatives were found to possess several pharmacological properties like antibacterial agents [8-10], including antiviral [11], antiallergic [12], hypoglycemic [13, 14] and diuretic [15].

A number of methods have been reported for the synthesis of pyrimidine derivatives [1625]. Here we report the synthesis of novel highly substituted pyrimidines bearing furanyl thiazole nucleous. The constitutions of all the products were confirmed using ${ }^{1} \mathrm{H}$ NMR, ${ }^{13} \mathrm{C}$ NMR, FTIR, and elemental analysis.

\section{EXPERIMENTAL}

Required all reagents were obtained commercially. Solvents were purified and dried before being used. All melting points were taken in open capillaries and are uncorrected. Thin-layer chromatography (TLC, on aluminium plates precoated with silica gel, $60 \mathrm{~F}_{254}, 0.25 \mathrm{~mm}$ thickness) (Merck, Darmstadt, Germany) was used for monitoring the progress of all reactions, purity and homogeneity of the synthesized compounds; eluent-hexane:ethyl acetate: (2:8). UV radiation and/or iodine were used as the visualizing agents. Elemental analysis $(\% \mathrm{C}, \mathrm{H}, \mathrm{N})$ was carried out by Perkin-Elmer 2400 series-II elemental analyzer (Perkin-Elmer, USA) and all compounds are within $\pm 0.4 \%$ of theory specified. The IR spectra were recorded in $\mathrm{KBr}$ on a Perkin-Elmer Spectrum GX FT-IR Spectrophotometer (Perkin-Elmer, USA) and only the characteristic peaks are reported in $\mathrm{cm}^{-1}$. ${ }^{1} \mathrm{H}$ NMR and ${ }^{13} \mathrm{C}$ NMR spectra were recorded in DMSO- $d_{6}$ on a Bruker Avance $400 \mathrm{~F}(\mathrm{MHz})$ 
spectrometer (Bruker Scientific Corporation Ltd., Switzerland) using solvent peak as internal standard at $400 \mathrm{MHz}$ and $100 \mathrm{MHz}$ respectively. Chemical shifts are reported in parts per million (ppm). Mass spectra were scanned on a Shimadzu LCMS 2010 spectrometer (Shimadzu, Tokyo, Japan).

\subsection{Synthesis of $N$-(4-(furan-2-yl)thiazol-2-yl)-4-methyl-3-oxopentanamide (2b)}

A mixture containing the 4-(furan-2-yl)thiazol-2-amine $1 \mathbf{a}(10 \mathrm{mmol})$, methyl isobutyrylacetate (10 $\mathrm{mmol})$, and catalytic amount of potassium hydroxide lie $(10 \%)$ was reflux at $110^{\circ} \mathrm{C}$ for the approximately 16-18 $\mathrm{h}$. The reaction was monitored by TLC. After completion of reaction, the solvent was removed under vaccuo when the reaction was completed. The solid or oil was crystallized from methanol to give pure product $\mathbf{2 b}$.

\subsection{Synthesis of ketene dithioacetals 3c.}

A $100 \mathrm{~mL}$ conical flask equipped with magnetic stirrer and septum was charged with a solution of $\mathrm{N}$-(4-(furan-2-yl)thiazol-2-yl)-4-methyl-3-oxopentanamide $\mathbf{2 b},(10 \mathrm{mmol})$ in DMF $(10 \mathrm{~mL})$. Dried $\mathrm{K}_{2} \mathrm{CO}_{3}(10 \mathrm{mmol})$ was added and the mixture was stirred for $2 \mathrm{~h}$ at room temperature. $\mathrm{CS}_{2}(30$ mmol) was added and the mixture was stirred for an additional $2 \mathrm{~h}$ at room temperature. Methyl iodide $\left(20 \mathrm{mmol}\right.$ ) was added at $0-5{ }^{\circ} \mathrm{C}$ and the mixture was stirred for $4 \mathrm{~h}$ before being poured onto water $(40 \mathrm{~mL})$. The precipitated crude product was purified by filtration followed by crystallization from EtOH. When the product was oil, the organic phase was extracted with $\mathrm{Et}_{2} \mathrm{O}(3 \times 10 \mathrm{~mL})$. The combined organic extracts were washed with $\mathrm{H}_{2} \mathrm{O}(2 \times 10 \mathrm{~mL})$, dried $\left(\mathrm{MgSO}_{4}\right)$, and concentrated in vaccuo to afford ketene dithioacetals directly used for the next step.

\subsection{General procedure for the synthesis of substituted pyrimidines $4 \mathrm{~d}$}

To a well stirred mixture of guanidine nitrate $(10 \mathrm{mmol})$ and sodium methoxide or sodium ethoxide (20 mmol) in methanol or ethanol was added the solution of ketene dithioacetals $\mathbf{3 c}(10 \mathrm{mmol})$ in methanol or ethanol within 10-15 min. The resulting reaction mixture was further stirred at $\mathrm{rt}$ for 15 min. then, reflux the reaction mixtures for $7 \mathrm{~h}$ on water bath. After completion of the reaction, the mixture was poured onto ice cold water. Thus, the obtained solid was filtered, wash with water and dried it and crystallization from EtOH to afford analytically pure products 4d.

\subsection{General procedure for the synthesis of substituted pyrimidines 5(a-t)}

To a well stirred mixture of substituted pyrimidines $\mathbf{4 d}(10 \mathrm{mmol})$ and piperidine $(2$ drops $)$ in ethanol $(10 \mathrm{~mL})$ was added various aromatic aldehyde $(10 \mathrm{mmol})$. The resulting reaction mixture was further stirred at $\mathrm{rt}$ for $15 \mathrm{~min}$. then, reflux the reaction mixtures for $4-5 \mathrm{~h}$ on water bath. After completion of the reaction, the mixture was poured onto ice cold water. Thus, the obtained solid was filtered, wash with water and dried it and crystallization from $\mathrm{MeOH}$ to afford analytically pure products $\mathbf{5}(\mathbf{a}-\mathbf{t})$. 


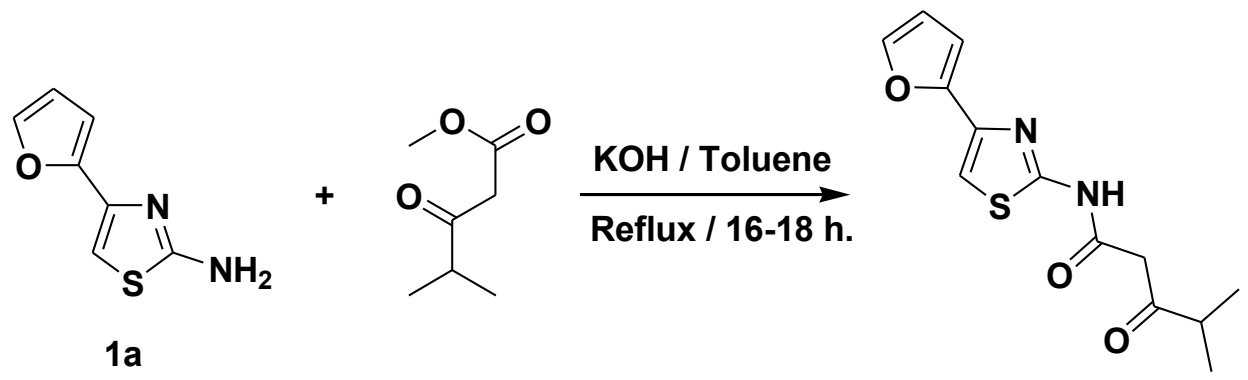

2b

Scheme 1 Synthetic pathway for the synthesis of $\mathbf{2 b}$<smiles>CC(C)C(=O)CC(=O)Nc1nc(-c2ccco2)cs1</smiles>

2b<smiles>CSC(SC)=C(C(=O)Nc1nc(-c2ccco2)cs1)C(=O)C(C)C</smiles>

$3 c$

Scheme 2 Synthetic pathway for the synthesis of $3 c$<smiles>CSC(SC)=C(C(=O)Nc1nc(-c2ccco2)cs1)C(=O)C(C)C</smiles>

$3 c$<smiles>[R]OC(=O)CCC(N)=O</smiles>

Where $\mathrm{R}=\mathrm{CH}_{3} / \mathrm{C}_{2} \mathrm{H}_{5}$<smiles>[R]Oc1nc(N)nc(C(C)C)c1C(=O)Nc1nc(-c2ccco2)cs1</smiles>

4d

Scheme 3 Synthetic pathway for the synthesis of $\mathbf{4 d}$

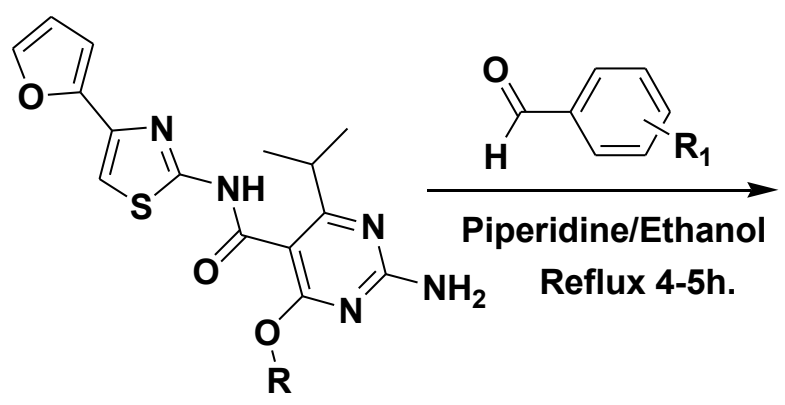<smiles>c1coc(-c2cscn2)c1</smiles>

4d

Where $\mathrm{R}_{1}=\mathrm{H}, \mathrm{CH}_{3}, \mathrm{OCH}_{3}, \mathrm{NO}_{2}, \mathrm{~F}, \mathrm{Cl}, \mathrm{Br}$

Scheme 4 Synthetic pathway for the synthesis of $5(a-t)$ 

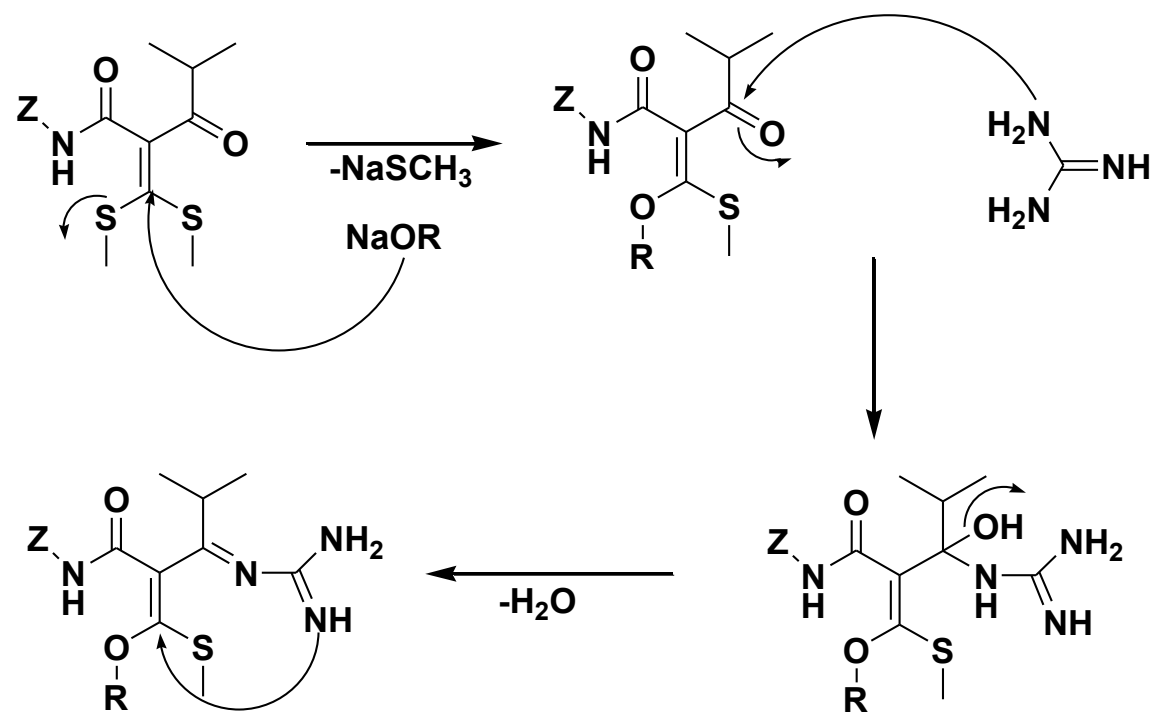

$-\mathrm{SHCH}_{3}$<smiles>[Z]NC(=O)c1c(O[R])nc(N)nc1C(C)C</smiles>

Where $\mathrm{R}=\mathrm{CH}_{3}, \mathrm{C}_{2} \mathrm{H}_{5}$

$\mathrm{Z}=$ Aryl Heterocycle

Scheme 5 Mechanism for the synthesis of pyrimidine derivatives

Table 1 Synthesis of substituted pyrimidines $5(\mathrm{a}-\mathrm{t})$

\begin{tabular}{|c|c|c|c|c|c|}
\hline Entry & $\mathbf{R}$ & $\mathbf{R}_{\mathbf{1}}$ & $\mathbf{R T}(\mathbf{h})$. & Yield \% & mp ${ }^{\mathbf{0}} \mathbf{C}$ \\
\hline $5 \mathrm{a}$ & $\mathrm{CH}_{3}$ & $\mathrm{H}$ & 5.0 & 80 & $150-152$ \\
\hline $5 \mathrm{~b}$ & $\mathrm{CH}_{3}$ & $4-\mathrm{CH}_{3}$ & 4.6 & 85 & $162-164$ \\
\hline $5 \mathrm{c}$ & $\mathrm{CH}_{3}$ & $3-\mathrm{CH}_{3}$ & 4.7 & 83 & $138-140$ \\
\hline $5 \mathrm{~d}$ & $\mathrm{CH}_{3}$ & $4-\mathrm{OCH}_{3}$ & 4.2 & 86 & $145-147$ \\
\hline $5 \mathrm{e}$ & $\mathrm{CH}_{3}$ & $4-\mathrm{NO}_{2}$ & 4.0 & 90 & $168-170$ \\
\hline $5 \mathrm{f}$ & $\mathrm{CH}_{3}$ & $3-\mathrm{NO}_{2}$ & 4.3 & 88 & $161-163$ \\
\hline $5 \mathrm{~g}$ & $\mathrm{CH}_{3}$ & $4-\mathrm{F}$ & 4.6 & 84 & $151-153$ \\
\hline $5 \mathrm{~h}$ & $\mathrm{CH}_{3}$ & $4-\mathrm{Br}$ & 4.8 & 82 & $139-141$ \\
\hline $5 \mathrm{i}$ & $\mathrm{CH}_{3}$ & $4-\mathrm{Cl}$ & 4.8 & 83 & $169-171$ \\
\hline $5 \mathrm{j}$ & $\mathrm{CH}_{3}$ & $3-\mathrm{Cl}$ & 4.9 & 77 & $148-150$ \\
\hline $5 \mathrm{k}$ & $\mathrm{C}_{2} \mathrm{H}_{5}$ & $\mathrm{H}$ & 4.9 & 81 & $139-141$ \\
\hline $5 \mathrm{l}$ & $\mathrm{C}_{2} \mathrm{H}_{5}$ & $4-\mathrm{CH}$ & 4.5 & 83 & $153-155$ \\
\hline $5 \mathrm{~m}$ & $\mathrm{C}_{2} \mathrm{H}_{5}$ & $3-\mathrm{CH}$ & 4.7 & 80 & $164-166$ \\
\hline $5 \mathrm{n}$ & $\mathrm{C}_{2} \mathrm{H}_{5}$ & $4-\mathrm{OCH}$ & 4.3 & 88 & $180-182$ \\
\hline $5 \mathrm{o}$ & $\mathrm{C}_{2} \mathrm{H}_{5}$ & $4-\mathrm{NO}_{2}$ & 4.0 & 89 & $175-177$ \\
\hline $5 \mathrm{p}$ & $\mathrm{C}_{2} \mathrm{H}_{5}$ & $3-\mathrm{NO}_{2}$ & 4.4 & 87 & $159-161$ \\
\hline $5 \mathrm{q}$ & $\mathrm{C}_{2} \mathrm{H}_{5}$ & $4-\mathrm{F}$ & 4.5 & 85 & $179-181$ \\
\hline $5 \mathrm{r}$ & $\mathrm{C}_{2} \mathrm{H}_{5}$ & $4-\mathrm{Br}$ & 4.9 & 80 & $183-185$ \\
\hline $5 \mathrm{~s}$ & $\mathrm{C}_{2} \mathrm{H}_{5}$ & $4-\mathrm{Cl}_{2}$ & 4.7 & 79 & $173-175$ \\
\hline $5 \mathrm{t}$ & $\mathrm{C}_{2} \mathrm{H}_{5}$ & $3-\mathrm{Cl}$ & 4.9 & 76 & $158-160$ \\
\hline
\end{tabular}


The required 4-(furan-2-yl)thiazol-2-amine 1a was prepared by solid phase reaction according to literature procedure [26].

In the current study, all the pyrimidine derivatives 5(a-t) were obtained in good yields by the reaction of various aromatic aldehydes and substituted amino pyrimidines $\mathbf{4 d}$ in ethanol, using piperidine as catalyst, at reflux temperature.(Scheme 4). The mechanism (Scheme 5), in ketene dithioacetal system the carbonyl carbon and $\beta$-carbon atoms regarded as hard and soft electrophilic centers, since the carbonyl carbon is adjacent to the hard-base oxygen while the $\beta$-carbon is flanked by the soft-base methylthio groups. Thus, the binucleophile guanidine in the presence of base attacks on carbonyl carbon of systems and formed substituted amino pyrimidine 4d product by removal of water molecule followed by methylthio as good leaving group.

The identity of the product determined by ${ }^{1} \mathrm{H}$ NMR, ${ }^{13} \mathrm{C}$ NMR, FT-IR spectral data, and molecular weight of some selected compounds were confirmed by mass spectrometry. ${ }^{1} \mathrm{H}$ NMR (DMSO-d6) spectrum of 5a, molecule of interest, exhibited doublet peak at $\delta 1.103-1.189 \mathrm{ppm}$ appeared for methyl protons of isopropyl group. Also exhibited multiplet at $\delta 3.223-3.202 \mathrm{ppm}$ appeared for methine proton and singlet at $\delta 3.530 \mathrm{ppm}$ of aromatic methoxy group. Aromatic protons as multiplets appeared at around $\delta 6.845-7.562 \mathrm{ppm}$ Moreover, it exhibited two singlet peak $\delta 9.139 \mathrm{ppm}$ appeared for methine proton and $\delta 10.288 \mathrm{ppm}$ appeared for $-\mathrm{NH}-$ proton. The ${ }^{13} \mathrm{C}$ NMR spectrum is in consonance with the structure assigned. In the ${ }^{13} \mathrm{C} N M R$ spectra, signals around $\delta 163.20 \mathrm{ppm}$ are attributed to carbonyl carbons and signals around $\delta 110.12-141.30 \mathrm{ppm}$ are attributed to aromatic carbons of compound 5a. Moreover 5a exhibited a distinctive signal at $\delta$ $56.20 \mathrm{ppm}$ for aromatic methoxy carbon, at $\delta 30.65 \mathrm{ppm}$ for methine carbon, at $\delta 21.36 \mathrm{ppm}$ for methyl carbon. The IR spectrum of compound 5a exhibited characteristic absorption band at 3,326 $\mathrm{cm}^{-1}$ for $-\mathrm{NH}-$ group. While $3,045 \mathrm{~cm}^{-1}$ for aromatic $\mathrm{C}-\mathrm{H}$ stretching and $1,688 \mathrm{~cm}^{-1}$ for carbonyl $\mathrm{C}=\mathrm{O}$ stretching. The mass spectra of compounds $\mathbf{5 a}$ and $\mathbf{5 k}$, molecules of interest, detected the expected molecular ion signals corresponding to respective molecular formula, i.e., mass spectra of compounds 5a and 5k gave molecular ion peak at $\mathrm{m} / \mathrm{z} 447.1(\mathrm{M}+1)$ and $\mathrm{m} / \mathrm{z} 461.1(\mathrm{M}+1)$ corresponding to molecular formula $\mathrm{C}_{23} \mathrm{H}_{21} \mathrm{~N}_{5} \mathrm{O}_{3} \mathrm{~S}$ and $\mathrm{C}_{24} \mathrm{H}_{23} \mathrm{~N}_{5} \mathrm{O}_{3} \mathrm{~S}$. The obtained elemental analysis values are in good agreement with theoretical data. Similarly, all these compounds were characterized on the basis of spectral studies. All spectroscopic data have been given in spectral data.

\section{SPECTRAL DATA}

(E)-2-(benzylideneamino)- $N$-(4-(furan-2-yl)thiazol-2-yl)-4-isopropyl methoxypyrimidine-5carboxamide (5a)

Yellow solid, yield 80\%, m.p. 150-152 ${ }^{\circ} \mathrm{C}$, IR (KBr, v, $\left.\mathrm{cm}^{-1}\right): 3326$ (N-H Str.), 3045 (Ar C-H Str.), 1688 (-C=O- Str.), ${ }^{1} \mathrm{H}$ NMR (400 MHz, DMSO- $\left.d_{6}\right) \delta_{\mathrm{H}}(\mathrm{ppm}): \delta 1.103-1.189\left(\mathrm{~d}, 6 \mathrm{H},-\mathrm{CH}\left(\mathrm{CH}_{3}\right)_{2}\right)$, 3.223-3.302 (m, 1H, - $\left.\underline{\mathrm{H}}\left(\mathrm{CH}_{3}\right)_{2}\right), 3.530\left(\mathrm{~s}, 3 \mathrm{H}, \mathrm{Ar}-\mathrm{OCH}_{3}\right), 6.845-7.562(\mathrm{~m}, 9 \mathrm{H}, \mathrm{Ar}-\mathrm{H}), 9.139$ (s, $1 \mathrm{H}, \mathrm{CH}), 10.288(\mathrm{~s}, 1 \mathrm{H}, \mathrm{NH}) .{ }^{13} \mathrm{C}$ NMR $\left(100 \mathrm{MHz}, \mathrm{DMSO}-d_{6}\right) \delta_{\mathrm{C}}(\mathrm{ppm}): 21.36\left(\underline{\mathrm{CH}}_{3}-\mathrm{CH}-\underline{\mathrm{CH}}_{3}\right)$, $30.65\left(\mathrm{CH}_{3}-\underline{\mathrm{CH}}-\mathrm{CH}_{3}\right), 56.20\left(\mathrm{Ar}-\mathrm{OCH}_{3}\right), 110.12,111.10,112.05,114.00,115.60,117.28,119.00$, $121.20,124.30,126.12,128.00,130.13,132.45,134.56,136.18,139.20,140.23,141.30$ (Ar-C), 163.20 (-CO-NH-). $\mathrm{MS}\left(\mathrm{M}^{+}\right): 447.14$, Anal. Calcd. for $\mathrm{C}_{23} \mathrm{H}_{21} \mathrm{~N}_{5} \mathrm{O}_{3} \mathrm{~S}(447.51): \mathrm{C} 61.73, \mathrm{H} 4.73, \mathrm{~N}$ 15.65 Found: C 61.20, H 4.11, N 15.70\%.

\section{(E)-2-(4-methylbenzylideneamino)- $N$-(4-(furan-2-yl)thiazol-2-yl)-4-isopropyl methoxypyrimidine-5-carboxamide (5b)}

Yellow solid, yield 85\%, m.p. 162-164 ${ }^{\circ} \mathrm{C}$, IR (KBr, v, $\mathrm{cm}^{-1}$ ): 3343 (N-H Str.), 3035 (Ar C-H Str.), 1680 (-C=O- Str.), ${ }^{1} \mathrm{H}$ NMR (400 MHz, DMSO- $\left.d_{6}\right) \delta_{\mathrm{H}}(\mathrm{ppm}): \delta 1.101-1.190\left(\mathrm{~d}, 6 \mathrm{H},-\mathrm{CH}\left(\mathrm{CH}_{3}\right)_{2}\right)$, $2.200\left(\mathrm{~s}, 3 \mathrm{H}, \mathrm{Ar}-\mathrm{CH}_{3}\right), 3.100-3.200\left(\mathrm{~m}, 1 \mathrm{H},-\mathrm{CH}\left(\mathrm{CH}_{3}\right)_{2}\right), 3.600\left(\mathrm{~s}, 3 \mathrm{H}, \mathrm{Ar}-\mathrm{OCH}_{3}\right), 6.721-7.213(\mathrm{~m}$, 8H, Ar-H), $9.100(\mathrm{~s}, 1 \mathrm{H}, \mathrm{CH}), 10.113(\mathrm{~s}, 1 \mathrm{H}, \mathrm{NH}) .{ }^{13} \mathrm{C}$ NMR $\left(100 \mathrm{MHz}, \mathrm{DMSO}-d_{6}\right) \delta_{\mathrm{C}}(\mathrm{ppm})$ : $21.15\left(\underline{\mathrm{CH}}_{3}-\mathrm{CH}-\underline{\mathrm{CH}}{ }_{3}\right), 24.20\left(\mathrm{Ar}-\underline{\mathrm{C}} \mathrm{H}_{3}\right), 30.20\left(\mathrm{CH}_{3}-\underline{\mathrm{C}} \mathrm{H}-\mathrm{CH}_{3}\right), 55.20\left(\mathrm{Ar}-\mathrm{O}_{\underline{C H}}\right), 108.13,109.12$, 
$112.00,113.45,114.26,116.13,117.64,112.12,122.36,124.22,125.20,127.44,129.14,131.28$,

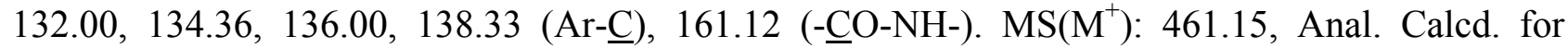
$\mathrm{C}_{24} \mathrm{H}_{23} \mathrm{~N}_{5} \mathrm{O}_{3} \mathrm{~S}$ (461.54): C 62.46, H 5.02, N 15.17 Found: C 62.20, H 4.80, N 15.18\%.

\section{(E)-2-(3-methylbenzylideneamino)- $N$-(4-(furan-2-yl)thiazol-2-yl)-4-isopropyl methoxypyrimidine-5-carboxamide $(5 \mathrm{c})$}

Yellow solid, yield 83\%, m.p. 138-140 C, IR (KBr, v, $\mathrm{cm}^{-1}$ ): 3388 (N-H Str.), 3060 (Ar C-H Str.), 1626 (-C=O- Str.), ${ }^{1} \mathrm{H}$ NMR (400 MHz, DMSO- $\left.d_{6}\right) \delta_{\mathrm{H}}(\mathrm{ppm}): \delta 1.236-1.300\left(\mathrm{~d}, 6 \mathrm{H},-\mathrm{CH}\left(\mathrm{CH}_{3}\right)_{2}\right)$, $2.288\left(\mathrm{~s}, 3 \mathrm{H}, \mathrm{Ar}-\mathrm{CH}_{3}\right), 3.223-3.300\left(\mathrm{~m}, 1 \mathrm{H},-\mathrm{CH}\left(\mathrm{CH}_{3}\right)_{2}\right), 3.700\left(\mathrm{~s}, 3 \mathrm{H}, \mathrm{Ar}-\mathrm{OCH}_{3}\right), 6.945-7.813(\mathrm{~m}$, $8 \mathrm{H}, \mathrm{Ar}-\mathrm{H}), 9.212(\mathrm{~s}, 1 \mathrm{H}, \mathrm{CH}), 10.313(\mathrm{~s}, 1 \mathrm{H}, \mathrm{NH}) .{ }^{13} \mathrm{C}$ NMR $\left(100 \mathrm{MHz}, \mathrm{DMSO}-d_{6}\right) \delta_{\mathrm{C}}(\mathrm{ppm})$ : $20.36\left(\underline{\mathrm{CH}}_{3}-\mathrm{CH}-\underline{\mathrm{CH}}_{3}\right), 24.90\left(\mathrm{Ar}-\underline{\mathrm{CH}}_{3}\right), 31.20\left(\mathrm{CH}_{3}-\underline{\mathrm{CH}}-\mathrm{CH}_{3}\right), 56.00\left(\mathrm{Ar}-\mathrm{OCH}_{3}\right), 109.15,110.02$, $112.03,113.23,115.00,116.32,117.23,118.20,120.45,123.40,125.20,128.30,130.22,132.25$, 135.21, 137.40, 140.11, 142.00 (Ar-ㅡ), 162.30 (-CO-NH-). $\mathrm{MS}\left(\mathrm{M}^{+}\right)$: 461.15, Anal. Calcd. for $\mathrm{C}_{24} \mathrm{H}_{23} \mathrm{~N}_{5} \mathrm{O}_{3} \mathrm{~S}$ (461.54): C 62.46, H 5.02, N 15.17 Found: C 62.20, H 4.80, N 15.18\%.

\section{(E)-2-(4-methoxybenzylideneamino)- $N$-(4-(furan-2-yl)thiazol-2-yl)-4-isopropyl} methoxypyrimidine-5-carboxamide (5d)

Yellow solid, yield 86\%, m.p. 145-147 C, IR (KBr, v, $\mathrm{cm}^{-1}$ ): 3339 (N-H Str.), 3012 (Ar C-H Str.), 1636 (-C=O- Str.), ${ }^{1} \mathrm{H}$ NMR (400 MHz, DMSO- $\left.d_{6}\right) \delta_{\mathrm{H}}(\mathrm{ppm}): \delta 1.080-1.125\left(\mathrm{~d}, 6 \mathrm{H},-\mathrm{CH}\left(\mathrm{CH}_{3}\right)_{2}\right)$, 33.125-3.206 (m, $\left.1 \mathrm{H},-\mathrm{C} \underline{\mathrm{H}}\left(\mathrm{CH}_{3}\right)_{2}\right), 3.589$ (s, 3H, $\left.\mathrm{Ar}-\mathrm{OCH}_{3}\right), 3.656$ (s, 3H, Ar- $\left.\mathrm{OCH}_{3}\right), 7.230-8.120$ $(\mathrm{m}, 8 \mathrm{H}, \mathrm{Ar}-\mathrm{H}), 9.118(\mathrm{~s}, 1 \mathrm{H}, \mathrm{CH}), 10.326(\mathrm{~s}, 1 \mathrm{H}, \mathrm{NH}) .{ }^{13} \mathrm{C} \mathrm{NMR}\left(100 \mathrm{MHz}, \mathrm{DMSO}-d_{6}\right) \delta_{\mathrm{C}}(\mathrm{ppm})$ : $21.96\left(\underline{\mathrm{CH}}_{3}-\mathrm{CH}-\underline{\mathrm{CH}}_{3}\right), 31.00\left(\mathrm{CH}_{3}-\underline{\mathrm{CH}}-\mathrm{CH}_{3}\right), 55.30\left(\mathrm{Ar}-\mathrm{O} \underline{\mathrm{CH}}{ }_{3}\right), 56.90\left(\mathrm{Ar}-\mathrm{O}_{\underline{C H}}\right), 110.12,111.20$, $113.00,114.36,115.30,117.00,119.30,121.03,122.00,124.56,126.30,127.00,129.54,131.02$, 133.00, 135.26, 137.18, 139.00 (Ar- $\underline{\mathrm{C}}), 160.36$ (-COC-NH-). MS(M $\left.{ }^{+}\right)$: 477.15, Anal. Calcd. for $\mathrm{C}_{24} \mathrm{H}_{23} \mathrm{~N}_{5} \mathrm{O}_{4} \mathrm{~S}$ (477.54): C 60.36, H 4.85, N 14.67 Found: C 60.50, H 4.23, N 14.40\%.

\section{(E)-2-(4-nitrobenzylideneamino)- $N$-(4-(furan-2-yl)thiazol-2-yl)-4-isopropyl methoxypyrimidine-5-carboxamide (5e)}

Yellow solid, yield 90\%, m.p. 168-170 C, IR (KBr, v, $\mathrm{cm}^{-1}$ ): 3351 (N-H Str.), 3036 (Ar C-H Str.), 1681 (-C=O- Str.), ${ }^{1} \mathrm{H}$ NMR (400 MHz, DMSO- $\left.d_{6}\right) \delta_{\mathrm{H}}(\mathrm{ppm}): \delta 1.126-1.256\left(\mathrm{~d}, 6 \mathrm{H},-\mathrm{CH}\left(\mathrm{CH}_{3}\right)_{2}\right)$, 3.125-3.258 (m, 1H, - $\left.\underline{\mathrm{H}}\left(\mathrm{CH}_{3}\right)_{2}\right), 3.623\left(\mathrm{~s}, 3 \mathrm{H}, \mathrm{Ar}-\mathrm{OCH}_{3}\right), 6.989-7.845$ (m, 8H, Ar-H), 9.165 (s, $1 \mathrm{H}, \mathrm{CH}), 10.126(\mathrm{~s}, 1 \mathrm{H}, \mathrm{NH}) .{ }^{13} \mathrm{C}$ NMR $\left(100 \mathrm{MHz}, \mathrm{DMSO}-d_{6}\right) \delta_{\mathrm{C}}(\mathrm{ppm}): 22.26\left(\underline{\mathrm{CH}}_{3}-\mathrm{CH}-\underline{\mathrm{CH}}_{3}\right)$, $30.26\left(\mathrm{CH}_{3}-\mathrm{CH}-\mathrm{CH}_{3}\right), 56.36\left(\mathrm{Ar}-\mathrm{OCH}_{3}\right), 111.02,112.00,113.36,114.25,116.65,117.00,118.30$, $120.00,122.00,124.36,126.30,127.32,128.54,130.12,132.25,134.61,135.00,138.14$ (Ar-C), 162.36 (-CO-NH-). MS(M $\left.{ }^{+}\right): 492.12$, Anal. Calcd. for $\mathrm{C}_{23} \mathrm{H}_{20} \mathrm{~N}_{6} \mathrm{O}_{5} \mathrm{~S}$ (492.51): C 56.09, $\mathrm{H} 4.09, \mathrm{~N}$ 17.06 Found: C 56.15, H 4.20, N 17.01\%.

\section{(E)-2-(3-nitrobenzylideneamino)- $N$-(4-(furan-2-yl)thiazol-2-yl)-4-isopropyl methoxypyrimidine-5-carboxamide (5f)}

Yellow solid, yield 88\%, m.p. 161-163으, IR (KBr, v, $\mathrm{cm}^{-1}$ ): 3377 (N-H Str.), 3042 (Ar C-H Str.), $1662(-\mathrm{C}=\mathrm{O}-\mathrm{Str}),.{ }^{1} \mathrm{H}$ NMR $\left(400 \mathrm{MHz}, \mathrm{DMSO}-d_{6}\right) \delta_{\mathrm{H}}(\mathrm{ppm}): \delta 1.205-1.306\left(\mathrm{~d}, 6 \mathrm{H},-\mathrm{CH}\left(\mathrm{CH}_{3}\right)_{2}\right)$, 3.263-3.299 (m, 1H, - $\left.\underline{\mathrm{H}}\left(\mathrm{CH}_{3}\right)_{2}\right), 3.626\left(\mathrm{~s}, 3 \mathrm{H}, \mathrm{Ar}-\mathrm{OCH}_{3}\right), 6.366-7.526(\mathrm{~m}, 8 \mathrm{H}, \mathrm{Ar}-\mathrm{H}), 9.188(\mathrm{~s}$, $1 \mathrm{H}, \mathrm{CH}), 10.313(\mathrm{~s}, 1 \mathrm{H}, \mathrm{NH}) .{ }^{13} \mathrm{C}$ NMR $\left(100 \mathrm{MHz}, \mathrm{DMSO}-d_{6}\right) \delta_{\mathrm{C}}(\mathrm{ppm}): 20.30\left(\underline{\mathrm{CH}}_{3}-\mathrm{CH}-\underline{\mathrm{CH}}_{3}\right)$, $31.24\left(\mathrm{CH}_{3}-\underline{\mathrm{C} H}-\mathrm{CH}_{3}\right), 56.21\left(\mathrm{Ar}-\mathrm{OCH}_{3}\right), 112.00,113.00,114.54,116.03,117.18,119.20,120.15$, $122.00,123.63,125.00,127.18,129.30,131.44,132.56,133.00,135.18,138.26,140.01$ (Ar-C), 162.60 (-CO-NH-). MS(M $\left.{ }^{+}\right): 492.12$, Anal. Calcd. for $\mathrm{C}_{23} \mathrm{H}_{20} \mathrm{~N}_{6} \mathrm{O}_{5} \mathrm{~S}$ (492.51): C 56.09, $\mathrm{H} 4.09, \mathrm{~N}$ 17.06 Found: C 56.15, H 4.20, N 17.01\%. 


\section{(E)-2-(4-fluorobenzylideneamino)- $N$-(4-(furan-2-yl)thiazol-2-yl)-4-isopropyl methoxypyrimidine-5-carboxamide (5g)}

Yellow solid, yield 84\%, m.p. 151-153 ${ }^{\circ} \mathrm{C}$, IR (KBr, v, $\left.\mathrm{cm}^{-1}\right)$ : 3380 (N-H Str.), 3030 (Ar C-H Str.), 1676 (-C=O- Str.), ${ }^{1} \mathrm{H}$ NMR (400 MHz, DMSO- $\left.d_{6}\right) \delta_{\mathrm{H}}(\mathrm{ppm}): \delta 1.236-1.300\left(\mathrm{~d}, 6 \mathrm{H},-\mathrm{CH}\left(\mathrm{CH}_{3}\right)_{2}\right)$, 3.120-3.201 (m, 1H, - $\left.\underline{\mathrm{H}}\left(\mathrm{CH}_{3}\right)_{2}\right), 3.712\left(\mathrm{~s}, 3 \mathrm{H}, \mathrm{Ar}-\mathrm{OCH}_{3}\right), 7.120-8.112(\mathrm{~m}, 8 \mathrm{H}, \mathrm{Ar}-\mathrm{H}), 9.201(\mathrm{~s}$, $1 \mathrm{H}, \mathrm{CH}), 10.306(\mathrm{~s}, 1 \mathrm{H}, \mathrm{NH}) .{ }^{13} \mathrm{C}$ NMR $\left(100 \mathrm{MHz}, \mathrm{DMSO}-d_{6}\right) \delta_{\mathrm{C}}(\mathrm{ppm}): 22.00\left(\underline{\mathrm{CH}}_{3}-\mathrm{CH}-\underline{\mathrm{C}} \mathrm{H}_{3}\right)$, $31.56\left(\mathrm{CH}_{3}-\underline{\mathrm{C}} \mathrm{H}-\mathrm{CH}_{3}\right), 57.13\left(\mathrm{Ar}-\mathrm{O} \underline{C H}_{3}\right), 110.12,112.30,113.00,114.56,115.00,117.03,119.23$, $120.56,122.00,124.50,126.30,127.23,128.31,130.54,132.65,135.01,137.18,139.20$ (Ar-C), 159.18 (-COO-NH-). $\mathrm{MS}\left(\mathrm{M}^{+}\right)$: 465.13, Anal. Calcd. for $\mathrm{C}_{23} \mathrm{H}_{20} \mathrm{FN}_{5} \mathrm{O}_{3} \mathrm{~S}$ (465.50): C 59.34, $\mathrm{H} 4.33, \mathrm{~N}$ 15.04 Found: C 59.22, H 4.40, N 15.26\%.

(E)-2-(4-bromobenzylideneamino)- $N$-(4-(furan-2-yl)thiazol-2-yl)-4-isopropyl methoxypyrimidine-5-carboxamide (5h)

Yellow solid, yield 82\%, m.p. 139-141 ${ }^{\circ} \mathrm{C}$, IR (KBr, v, $\mathrm{cm}^{-1}$ ): 3360 (N-H Str.), 3078 (Ar C-H Str.), 1651 (-C=O- Str.), ${ }^{1} \mathrm{H}$ NMR (400 MHz, DMSO- $\left.d_{6}\right) \delta_{\mathrm{H}}(\mathrm{ppm}): \delta 1.165-1.206\left(\mathrm{~d}, 6 \mathrm{H},-\mathrm{CH}\left(\mathrm{CH}_{3}\right)_{2}\right)$, 3.212-3.314 (m, 1H, - $\left.\underline{\mathrm{H}}\left(\mathrm{CH}_{3}\right)_{2}\right), 3.623\left(\mathrm{~s}, 3 \mathrm{H}, \mathrm{Ar}-\mathrm{OCH}_{3}\right), 7.112-8.201(\mathrm{~m}, 8 \mathrm{H}, \mathrm{Ar}-\mathrm{H}), 9.213(\mathrm{~s}$, $1 \mathrm{H}, \mathrm{CH}), 10.400(\mathrm{~s}, 1 \mathrm{H}, \mathrm{NH}) .{ }^{13} \mathrm{C}$ NMR $\left(100 \mathrm{MHz}, \mathrm{DMSO}-d_{6}\right) \delta_{\mathrm{C}}(\mathrm{ppm}): 21.98\left(\underline{\mathrm{CH}}_{3}-\mathrm{CH}-\underline{\mathrm{CH}}_{3}\right)$, $31.25\left(\mathrm{CH}_{3}-\underline{\mathrm{C}} \mathrm{H}-\mathrm{CH}_{3}\right), 56.44\left(\mathrm{Ar}-\mathrm{OCH}_{3}\right), 109.56,110.23,112.00,115.30,116.32,118.00,120.11$, 121.56, 123.06, 125.66, 127.18, 129.40, 131.34, 133.00, 135.60, 137.18, 139.01, 142.00 (Ar-C ), 164.00 (-CO-NH-). $\mathrm{MS}\left(\mathrm{M}^{+}\right)$: 525.05, Anal. Calcd. for $\mathrm{C}_{23} \mathrm{H}_{20} \mathrm{BrN}_{5} \mathrm{O}_{3} \mathrm{~S}$ (526.41): C 52.48, H 3.83, N 13.30 Found: C 52.52, H 3.75, N 13.28\%.

\section{(E)-2-(4-chlorobenzylideneamino)- $N$-(4-(furan-2-yl)thiazol-2-yl)-4-isopropyl} methoxypyrimidine-5-carboxamide (5i)

Yellow solid, yield 83\%, m.p. 169-171 ${ }^{\circ} \mathrm{C}$, IR (KBr, v, $\mathrm{cm}^{-1}$ ): 3375 (N-H Str.), 3018 (Ar C-H Str.), 1668 (-C=O- Str.), ${ }^{1} \mathrm{H}$ NMR (400 MHz, DMSO- $\left.d_{6}\right) \delta_{\mathrm{H}}(\mathrm{ppm}): \delta 1.145-1.231\left(\mathrm{~d}, 6 \mathrm{H},-\mathrm{CH}\left(\mathrm{CH}_{3}\right)_{2}\right)$, 3.202-3.298 (m, 1H, $\left.-\mathrm{C} \underline{\mathrm{H}}\left(\mathrm{CH}_{3}\right)_{2}\right), 3.714\left(\mathrm{~s}, 3 \mathrm{H}, \mathrm{Ar}-\mathrm{OCH}_{3}\right), 6.982-7.858(\mathrm{~m}, 8 \mathrm{H}, \mathrm{Ar}-\mathrm{H}), 9.230(\mathrm{~s}$, $1 \mathrm{H}, \mathrm{CH}), 10.412(\mathrm{~s}, 1 \mathrm{H}, \mathrm{NH}) .{ }^{13} \mathrm{C}$ NMR $\left(100 \mathrm{MHz}, \mathrm{DMSO}-d_{6}\right) \delta_{\mathrm{C}}(\mathrm{ppm}): 20.13\left(\underline{\mathrm{CH}}_{3}-\mathrm{CH}-\underline{\mathrm{CH}}_{3}\right)$, $31.33\left(\mathrm{CH}_{3}-\underline{\mathrm{C}} \mathrm{H}-\mathrm{CH}_{3}\right), 56.90\left(\mathrm{Ar}-\mathrm{OCH}_{3}\right), 111.02,112.30,113.56,114.00,115.60,117.20,119.20$, 121.04, 122.36, 125.64, 127.34, 129.36, 131.02, 133.25, 135.41, 137.25, 139.56, 141.00 (Ar-C), 162.38 (-CO-NH-). $\mathrm{MS}\left(\mathrm{M}^{+}\right)$: 481.10, Anal. Calcd. for $\mathrm{C}_{23} \mathrm{H}_{20} \mathrm{ClN}_{5} \mathrm{O}_{3} \mathrm{~S}$ (481.95): C 57.32, H 4.18, N 14.53 Found: C 57.35, H 4.12, N 14.36\%

\section{(E)-2-(3-chlorobenzylideneamino)- $N$-(4-(furan-2-yl)thiazol-2-yl)-4-isopropyl} methoxypyrimidine-5-carboxamide $(5 \mathrm{j})$

Yellow solid, yield 77\%, m.p. 148-150 C, IR (KBr, v, $\mathrm{cm}^{-1}$ ): 3369 (N-H Str.), 3026 (Ar C-H Str.), 1686 (-C=O- Str.), ${ }^{1} \mathrm{H}$ NMR (400 MHz, DMSO- $\left.d_{6}\right) \delta_{\mathrm{H}}(\mathrm{ppm}): \delta 1.156-1.236\left(\mathrm{~d}, 6 \mathrm{H},-\mathrm{CH}\left(\mathrm{CH}_{3}\right)_{2}\right)$, 3.124-3.215 (m, 1H, - $\left.\underline{\mathrm{H}}\left(\mathrm{CH}_{3}\right)_{2}\right), 3.715\left(\mathrm{~s}, 3 \mathrm{H}, \mathrm{Ar}-\mathrm{OCH}_{3}\right), 6.898-7.568(\mathrm{~m}, 8 \mathrm{H}, \mathrm{Ar}-\mathrm{H}), 9.228(\mathrm{~s}$, $1 \mathrm{H}, \mathrm{CH}), 10.315(\mathrm{~s}, 1 \mathrm{H}, \mathrm{NH}) .{ }^{13} \mathrm{C}$ NMR $\left(100 \mathrm{MHz}, \mathrm{DMSO}-d_{6}\right) \delta_{\mathrm{C}}(\mathrm{ppm}): 22.01\left(\underline{\mathrm{CH}}_{3}-\mathrm{CH}-\underline{C H}_{3}\right)$, $29.98\left(\mathrm{CH}_{3}-\underline{\mathrm{C}} \mathrm{H}-\mathrm{CH}_{3}\right), 56.43\left(\mathrm{Ar}-\mathrm{O} \underline{\mathrm{C}} \mathrm{H}_{3}\right), 110.20,112.30,113.54,115.20,116.54,117.00,119.67$, 121.30, 122.00, 125.36, 127.40, 128.23, 129.00, 131.50, 133.00, 135.64, 137.80, 139.02 (Ar-C ), 163.00 (-CO-NH-). $\mathrm{MS}\left(\mathrm{M}^{+}\right): 481.10$, Anal. Calcd. for $\mathrm{C}_{23} \mathrm{H}_{20} \mathrm{ClN}_{5} \mathrm{O}_{3} \mathrm{~S}$ (481.95): C 57.32, H 4.18, N 14.53 Found: C 57.35, H 4.12, N 14.36\% 


\section{(E)-2-(benzylideneamino)-4-ethoxy- $N$-(4-(furan-2-yl)thiazol-2-yl)-6-isopropylpyrimidine-5- carboxamide (5k)}

Yellow solid, yield 81\%, m.p. 139-141 ${ }^{\circ} \mathrm{C}$, IR (KBr, v, $\mathrm{cm}^{-1}$ ): 3397 (N-H Str.), 3025 (Ar C-H Str.), 1652 (-C=O- Str.). ${ }^{1} \mathrm{H}$ NMR (400 MHz, DMSO- $\left.d_{6}\right) \delta_{\mathrm{H}}(\mathrm{ppm}): \delta 1.263-1.369\left(\mathrm{~d}, 6 \mathrm{H},-\mathrm{CH}\left(\mathrm{C}_{3}\right)_{2}\right)$, 1.312-1.398 (t, 3H, Ar-OCH $\left.{ }_{2}-\underline{C H}_{3}\right), 3.236-3.365$ (m, $\left.1 \mathrm{H},-\mathrm{CH}\left(\mathrm{CH}_{3}\right)_{2}\right)$, 3.912-3.980 (q, $2 \mathrm{H}, \mathrm{Ar}-$ $\left.\mathrm{OCH}_{2}-\mathrm{CH}_{3}\right), 6.563-7.569(\mathrm{~m}, 9 \mathrm{H}, \mathrm{Ar}-\mathrm{H}), 9.138(\mathrm{~s}, 1 \mathrm{H}, \mathrm{CH}), 10.326(\mathrm{~s}, 1 \mathrm{H}, \mathrm{NH}) .{ }^{13} \mathrm{C} \mathrm{NMR}(100$ $\left.\mathrm{MHz}, \mathrm{DMSO}-d_{6}\right) \delta_{\mathrm{C}}(\mathrm{ppm}): 17.13\left(\mathrm{Ar}-\mathrm{OCH}_{2}-\underline{\mathrm{CH}}_{3}\right), 21.20\left(\underline{\mathrm{CH}}_{3}-\mathrm{CH}-\underline{\mathrm{CH}}{ }_{3}\right), 31.56\left(\mathrm{CH}_{3}-\underline{\mathrm{C}} \mathrm{H}-\mathrm{CH}_{3}\right)$, $59.50\left(\mathrm{Ar}-\mathrm{OCH}_{2}-\mathrm{CH}_{3}\right), 113.25,114.32,115.34,117.18,119.20,121.00,123.00,125.36,126.30$, 129.20, 131.00, 133.02, 134.50, 136.21, 138.56, 139.30, 141.21, 142.00 (Ar-C ), 164.20 (-CO-NH-). $\mathrm{MS}\left(\mathrm{M}^{+}\right)$: 461.15, Anal. Calcd. for $\mathrm{C}_{24} \mathrm{H}_{23} \mathrm{~N}_{5} \mathrm{O}_{3} \mathrm{~S}$ (461.54): C 62.46, H 4.02, N 15.17 Found: $\mathrm{C}$ $62.56, \mathrm{H} 4.22$, N $15.10 \%$.

\section{(E)-2-(4-methylbenzylideneamino)-4-ethoxy- $N$-(4-(furan-2-yl)thiazol-2-yl)-6- isopropylpyrimidine-5-carboxamide (5l)}

Yellow solid, yield 83\%, m.p. 155-153 ${ }^{\circ} \mathrm{C}$, IR (KBr, v, $\mathrm{cm}^{-1}$ ): 3390 (N-H Str.), 3025 (Ar C-H Str.), 1650 (-C=O- Str.). ${ }^{1} \mathrm{H}$ NMR (400 MHz, DMSO- $\left.d_{6}\right) \delta_{\mathrm{H}}(\mathrm{ppm}): \delta 1.100-1.200\left(\mathrm{~d}, 6 \mathrm{H},-\mathrm{CH}\left(\mathrm{CH}_{3}\right)_{2}\right)$, 1.212-1.298 (t, $\left.3 \mathrm{H}, \mathrm{Ar}-\mathrm{OCH}_{2}-\mathrm{C}_{3}\right), 2.300\left(\mathrm{~s}, 3 \mathrm{H}, \mathrm{Ar}-\mathrm{CH}_{3}\right), 3.200-3.300\left(\mathrm{~m}, 1 \mathrm{H},-\mathrm{C} \underline{\mathrm{H}}\left(\mathrm{CH}_{3}\right)_{2}\right)$, 3.900-3.980 (q, 2H, Ar-OCH $\left.{ }_{2}-\mathrm{CH}_{3}\right), 6.500-7.650$ (m, 8H, Ar-H), 9.200 (s, 1H, CH), $10.213(\mathrm{~s}, 1 \mathrm{H}$, $\mathrm{NH}) .{ }^{13} \mathrm{C}$ NMR $\left(100 \mathrm{MHz}, \mathrm{DMSO}-d_{6}\right) \delta_{\mathrm{C}}(\mathrm{ppm}): 18.12\left(\mathrm{Ar}-\mathrm{OCH}_{2}-\underline{\mathrm{CH}}_{3}\right), 22.36\left(\underline{\mathrm{CH}}_{3}-\mathrm{CH}-\underline{\mathrm{CH}}_{3}\right)$, $25.36\left(\mathrm{Ar}-\mathrm{CH}_{3}\right), 31.28\left(\mathrm{CH}_{3}-\underline{\mathrm{C}} \mathrm{H}-\mathrm{CH}_{3}\right), 59.66\left(\mathrm{Ar}-\mathrm{OCH}_{2}-\mathrm{CH}_{3}\right), 109.23,110.16,113.24,114.00$, 115.36, 116.87, 118.00, 120.33, 122.00, 124.39, 126.36, 128.45, 130.12, 132.14, 133.00, 135.26, 138.12, 140.33 (Ar- $-\underline{C}$ ), 163.24 (-ㅡ- $-\mathrm{NH}-$ ). $\mathrm{MS}\left(\mathrm{M}^{+}\right)$: 475.17, Anal. Calcd. for $\mathrm{C}_{25} \mathrm{H}_{25} \mathrm{~N}_{5} \mathrm{O}_{3} \mathrm{~S}$ (475.56): C 63.14, H 5.30, N 14.73 Found: C 63.18, H 5.40, N 14.70\%.

\section{(E)-2-(3-methylbenzylideneamino)-4-ethoxy- $N$-(4-(furan-2-yl)thiazol-2-yl)-6-} isopropylpyrimidine-5-carboxamide $(5 \mathrm{~m})$

Yellow solid, yield 80\%, m.p. 164-166 ${ }^{\circ} \mathrm{C}$, IR (KBr, v, $\left.\mathrm{cm}^{-1}\right)$ : 3356 (N-H Str.), 3025 (Ar C-H Str.), $1636(-\mathrm{C}=\mathrm{O}-\mathrm{Str}.) .{ }^{1} \mathrm{H}$ NMR $\left(400 \mathrm{MHz}, \mathrm{DMSO}-d_{6}\right) \delta_{\mathrm{H}}(\mathrm{ppm}): \delta 1.135-1.188\left(\mathrm{~d}, 6 \mathrm{H},-\mathrm{CH}\left(\mathrm{CH}_{3}\right)_{2}\right)$, 1.200-1.290 (t, 3H, Ar-OCH $\left.-\mathrm{CH}_{3}\right), 2.420$ (s, 3H, Ar- $\left.\mathrm{CH}_{3}\right), 3.198-3.203\left(\mathrm{~m}, 1 \mathrm{H},-\mathrm{CH}\left(\mathrm{CH}_{3}\right)_{2}\right)$, 3.980-34.050 (q, 2H, Ar-OC $\left.\underline{H}_{2}-\mathrm{CH}_{3}\right), 6.890-7.456(\mathrm{~m}, 8 \mathrm{H}, \mathrm{Ar}-\mathrm{H}), 9.312(\mathrm{~s}, 1 \mathrm{H}, \mathrm{CH}), 10.525(\mathrm{~s}$, $1 \mathrm{H}, \mathrm{NH}) .{ }^{13} \mathrm{C} \mathrm{NMR}\left(100 \mathrm{MHz}, \mathrm{DMSO}-d_{6}\right) \delta_{\mathrm{C}}(\mathrm{ppm}): 17.88\left(\mathrm{Ar}_{-} \mathrm{OCH}_{2}-\underline{\mathrm{CH}}_{3}\right), 22.96\left(\underline{\mathrm{CH}}_{3}-\mathrm{CH}-\underline{\mathrm{CH}}_{3}\right)$, $25.30\left(\mathrm{Ar}-\mathrm{CH}_{3}\right), 31.90\left(\mathrm{CH}_{3}-\underline{\mathrm{C}} \mathrm{H}-\mathrm{CH}_{3}\right), 60.03\left(\mathrm{Ar}-\mathrm{OCH}_{2}-\mathrm{CH}_{3}\right), 110.15,112.20,113.36,115.41$, $116.20,117.18,119.80,121.00,123.02,125.00,127.13,129.36,131.24,133.02,135.56,137.14$,

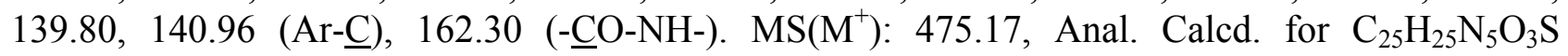
(475.56): C 63.14, H 5.30, N 14.73 Found: C 63.18, H 5.40, N 14.70\%.

\section{(E)-2-(4-methoxybenzylideneamino)-4-ethoxy- $N$-(4-(furan-2-yl)thiazol-2-yl)-6-} isopropylpyrimidine-5-carboxamide (5n)

Yellow solid, yield 88\%, m.p. 180-182 $\mathrm{C}$, IR (KBr, v, $\left.\mathrm{cm}^{-1}\right): 3369$ (N-H Str.), 3070 (Ar C-H Str.), 1688 (-C=O- Str.). ${ }^{1} \mathrm{H}$ NMR (400 MHz, DMSO- $\left.d_{6}\right) \delta_{\mathrm{H}}(\mathrm{ppm}): \delta 1.145-1.256\left(\mathrm{~d}, 6 \mathrm{H},-\mathrm{CH}\left(\mathrm{CH}_{3}\right)_{2}\right)$, 1.290-1.326 (t, 3H, Ar-OCH$\left.-\mathrm{CH}_{3}\right), 3.220-3.290\left(\mathrm{~m}, 1 \mathrm{H},-\mathrm{C} \underline{\mathrm{H}}\left(\mathrm{CH}_{3}\right)_{2}\right), 3.712\left(\mathrm{~s}, 3 \mathrm{H}, \mathrm{Ar}-\mathrm{OCH}_{3}\right)$, 3.826-3.900 (q, 2H, Ar-OCH$\left.{ }_{2}-\mathrm{CH}_{3}\right), 6.713-7.621(\mathrm{~m}, 8 \mathrm{H}, \mathrm{Ar}-\mathrm{H}), 9.288(\mathrm{~s}, 1 \mathrm{H}, \mathrm{CH}), 10.245(\mathrm{~s}, 1 \mathrm{H}$, $\mathrm{NH}) .{ }^{13} \mathrm{C}$ NMR $\left(100 \mathrm{MHz}, \mathrm{DMSO}-d_{6}\right) \delta_{\mathrm{C}}(\mathrm{ppm}): 18.33\left(\mathrm{Ar}-\mathrm{OCH}_{2}-\underline{\mathrm{CH}}_{3}\right), 23.00\left(\underline{\mathrm{CH}}_{3}-\mathrm{CH}-\underline{\mathrm{CH}}_{3}\right)$, $32.01\left(\mathrm{CH}_{3}-\underline{\mathrm{CH}}-\mathrm{CH}_{3}\right), 56.60\left(\mathrm{Ar}-\mathrm{OCH}_{3}\right), 59.03\left(\mathrm{Ar}-\mathrm{OCH}_{2}-\mathrm{CH}_{3}\right), 111.02,112.21,113.26,115.20$, $116.54,117.80,118.30,120.32,122.00,124.53,126.30,128.41,130.12,131.23,133.56,135.20$, 138.34, 139.21 (Ar- $\underline{\text { C) }), ~} 165.21$ (-CO-NH-). MS(M $\left.{ }^{+}\right)$: 491.16, Anal. Calcd. for $\mathrm{C}_{25} \mathrm{H}_{25} \mathrm{~N}_{5} \mathrm{O}_{4} \mathrm{~S}$ (491.56): C 61.08, H 5.13, N 14.25 Found: C 61.20, H 5.18, N 14.28\%. 
(E)-2-(4-nitrobenzylideneamino)-4-ethoxy- $N$-(4-(furan-2-yl)thiazol-2-yl)-6isopropylpyrimidine-5-carboxamide (50)

Yellow solid, yield 89\%, m.p. 175-177 ${ }^{\circ}$, IR (KBr, v, $\mathrm{cm}^{-1}$ ): 3380 (N-H Str.), 3076 (Ar C-H Str.), 1672 (-C=O- Str.). ${ }^{1} \mathrm{H}$ NMR (400 MHz, DMSO- $\left.d_{6}\right) \delta_{\mathrm{H}}(\mathrm{ppm}): \delta 1.145-1.236\left(\mathrm{~d}, 6 \mathrm{H},-\mathrm{CH}\left(\mathrm{CH}_{3}\right)_{2}\right)$, 1.300-1.356 (t, 3H, Ar-OCH $\left.{ }_{2}-\underline{C H}_{3}\right), 3.230-3.298$ (m, $\left.1 \mathrm{H},-\mathrm{CH}\left(\mathrm{CH}_{3}\right)_{2}\right)$, 3.890-3.980 (q, $2 \mathrm{H}, \mathrm{Ar}-$ $\left.\mathrm{OC}_{2}-\mathrm{CH}_{3}\right), 7.125-8.230(\mathrm{~m}, 8 \mathrm{H}, \mathrm{Ar}-\mathrm{H}), 9.321(\mathrm{~s}, 1 \mathrm{H}, \mathrm{CH}), 10.336(\mathrm{~s}, 1 \mathrm{H}, \mathrm{NH}) .{ }^{13} \mathrm{C}$ NMR $(100$ $\left.\mathrm{MHz}, \mathrm{DMSO}-d_{6}\right) \delta_{\mathrm{C}}(\mathrm{ppm}): 19.12\left(\mathrm{Ar}-\mathrm{OCH}_{2}-\underline{\mathrm{CH}}_{3}\right), 23.01\left(\underline{\mathrm{CH}}_{3}-\mathrm{CH}-\underline{\mathrm{CH}}{ }_{3}\right), 30.21\left(\mathrm{CH}_{3}-\underline{\mathrm{C}}-\mathrm{CH}_{3}\right)$, $58.30\left(\mathrm{Ar}-\mathrm{OCH}_{2}-\mathrm{CH}_{3}\right), 112.03,113.05,114.00,116.31,117.80,119.20,121.04,123.00,125.46$, 127.41, 129.50, 131.54, 133.00, 135.62, 137.41, 139.80, 140.13, 141.00 (Ar-C), 159.12 (-CO-NH-). $\mathrm{MS}\left(\mathrm{M}^{+}\right)$: 506.14, Anal. Calcd. for $\mathrm{C}_{24} \mathrm{H}_{22} \mathrm{~N}_{6} \mathrm{O}_{5} \mathrm{~S}$ (506.53): $\mathrm{C}$ 56.91, $\mathrm{H}$ 4.38, N 16.59 Found: $\mathrm{C}$ $56.85, \mathrm{H} 4.42$, N $16.62 \%$.

(E)-2-(3-nitrobenzylideneamino)-4-ethoxy- $N$-(4-(furan-2-yl)thiazol-2-yl)-6isopropylpyrimidine-5-carboxamide (5p)

Yellow solid, yield 87\%, m.p. 159-161 ${ }^{\circ} \mathrm{C}$, IR (KBr, v, $\left.\mathrm{cm}^{-1}\right): 3378$ (N-H Str.), 3056 (Ar C-H Str.), 1670 (-C=O- Str.). ${ }^{1} \mathrm{H}$ NMR (400 MHz, DMSO- $\left.d_{6}\right) \delta_{\mathrm{H}}(\mathrm{ppm}): \delta 1.203-1.280\left(\mathrm{~d}, 6 \mathrm{H},-\mathrm{CH}\left(\mathrm{CH}_{3}\right)_{2}\right)$, 1.320-1.356 (t, 3H, Ar-OCH $\left.2-\underline{C H}_{3}\right), 3.254-3.298\left(\mathrm{~m}, 1 \mathrm{H},-\mathrm{C} \underline{\mathrm{H}}\left(\mathrm{CH}_{3}\right)_{2}\right), 3.920-4.00$ (q, $2 \mathrm{H}, \mathrm{Ar}-$ $\left.\mathrm{OC}_{2}-\mathrm{CH}_{3}\right), 7.123-8.225(\mathrm{~m}, 8 \mathrm{H}, \mathrm{Ar}-\mathrm{H}), 9.312(\mathrm{~s}, 1 \mathrm{H}, \mathrm{CH}), 10.378(\mathrm{~s}, 1 \mathrm{H}, \mathrm{NH}) .{ }^{13} \mathrm{C} \mathrm{NMR}(100$ $\left.\mathrm{MHz}, \mathrm{DMSO}-d_{6}\right) \delta_{\mathrm{C}}(\mathrm{ppm}): 18.01\left(\mathrm{Ar}-\mathrm{OCH}_{2}-\underline{\mathrm{CH}}_{3}\right), 22.19\left(\underline{\mathrm{CH}}_{3}-\mathrm{CH}-\underline{\mathrm{CH}}_{3}\right), 31.98\left(\mathrm{CH}_{3}-\underline{\mathrm{C}}-\mathrm{H}_{-}-\mathrm{CH}_{3}\right)$, $60.01\left(\mathrm{Ar}-\mathrm{OCH}_{2}-\mathrm{CH}_{3}\right), 111.00,112.03,114.01,116.24,117.30,118.32,120.21,122.25,124.10$, 126.14, 127.30, 129.31, 131.20, 133.04, 135.24, 136.48, 138.30, 140.24 (Ar-C) ), 164.30 (-COO-NH-). $\mathrm{MS}\left(\mathrm{M}^{+}\right)$: 506.14, Anal. Calcd. for $\mathrm{C}_{24} \mathrm{H}_{22} \mathrm{~N}_{6} \mathrm{O}_{5} \mathrm{~S}$ (506.53): C 56.91, H 4.38, N 16.59 Found: C 56.85, H 4.42, N 16.62\%.

(E)-2-(4-fluorobenzylideneamino)-4-ethoxy- $N$-(4-(furan-2-yl)thiazol-2-yl)-6isopropylpyrimidine-5-carboxamide (5q)

Yellow solid, yield 85\%, m.p. 179-181 ${ }^{\circ} \mathrm{C}$, Anal. IR (KBr, v, $\mathrm{cm}^{-1}$ ): 3388 (N-H Str.), 3041 (Ar C-H Str.), 1646 (-C=O- Str.). ${ }^{1} \mathrm{H}$ NMR (400 MHz, DMSO-d $) \delta_{\mathrm{H}}(\mathrm{ppm}): \delta 1.189-1.212(\mathrm{~d}, 6 \mathrm{H}$, $\left.\mathrm{CH}\left(\mathrm{CH}_{3}\right)_{2}\right), 1.250-1.300$ (t, $\left.3 \mathrm{H}, \mathrm{Ar}-\mathrm{OCH}_{2}-\underline{\mathrm{CH}}_{3}\right), 3.198-3.256$ (m, $\left.1 \mathrm{H},-\mathrm{C} \underline{\mathrm{H}}\left(\mathrm{CH}_{3}\right)_{2}\right), 3.950-4.023$ (q, $\left.2 \mathrm{H}, \mathrm{Ar}-\mathrm{OCH} \underline{H}_{2}-\mathrm{CH}_{3}\right), 6.890-7.450(\mathrm{~m}, 8 \mathrm{H}, \mathrm{Ar}-\mathrm{H}), 9.313(\mathrm{~s}, 1 \mathrm{H}, \mathrm{CH}), 10.378(\mathrm{~s}, 1 \mathrm{H}, \mathrm{NH}) .{ }^{13} \mathrm{C} \mathrm{NMR}$ $\left(100 \mathrm{MHz}, \mathrm{DMSO}-d_{6}\right) \delta_{\mathrm{C}}(\mathrm{ppm}): 17.20\left(\mathrm{Ar}-\mathrm{OCH}_{2}-\underline{\mathrm{CH}_{3}}\right), 22.96\left(\underline{\mathrm{CH}}_{3}-\mathrm{CH}-\underline{\mathrm{CH}_{3}}\right), 32.00\left(\mathrm{CH}_{3}-\underline{\mathrm{CH}}-\right.$ $\left.\mathrm{CH}_{3}\right), 61.30\left(\mathrm{Ar}-\mathrm{OCH}_{2}-\mathrm{CH}_{3}\right), 110.23,112.03,113.24,115.00,117.31,119.20,121.04,123.65$, $125.40,127.41,129.50,131.02,133.06,135.40,137.50,139.00,140.12,142.05$ (Ar-C $), 165.00(-$ CO-NH-). $\mathrm{MS}\left(\mathrm{M}^{+}\right)$: 479.14, Calcd. for $\mathrm{C}_{24} \mathrm{H}_{22} \mathrm{FN}_{5} \mathrm{O}_{3} \mathrm{~S}$ (479.53): C 60.11, H 3.62, N 13.96 Found: $\mathrm{C}$ 59.95, H 3.64, N 13.80\%

(E)-2-(4-bromobenzylideneamino)-4-ethoxy- $N$-(4-(furan-2-yl)thiazol-2-yl)-6isopropylpyrimidine-5-carboxamide $(5 r)$

Yellow solid, yield 80\%, m.p. 183-185 C, IR (KBr, v, cm ${ }^{-1}$ ): 3388 (N-H Str.), 3025 (Ar C-H Str.), 1684 (-C=O- Str.). ${ }^{1} \mathrm{H}$ NMR (400 MHz, DMSO- $\left.d_{6}\right) \delta_{\mathrm{H}}(\mathrm{ppm}): \delta 1.201-1.250\left(\mathrm{~d}, 6 \mathrm{H},-\mathrm{CH}\left(\mathrm{CH}_{3}\right)_{2}\right)$, 1.300-1.326 (t, 3H, Ar-OCH$\left.-\mathrm{CH}_{3}\right), 3.200-3.300\left(\mathrm{~m}, 1 \mathrm{H},-\mathrm{CH}\left(\mathrm{CH}_{3}\right)_{2}\right), 3.856-3.900(\mathrm{q}, 2 \mathrm{H}, \mathrm{Ar}-$ $\left.\mathrm{OCH}_{2}-\mathrm{CH}_{3}\right), 7.450-8.500(\mathrm{~m}, 8 \mathrm{H}, \mathrm{Ar}-\mathrm{H}), 9.321(\mathrm{~s}, 1 \mathrm{H}, \mathrm{CH}), 10.188(\mathrm{~s}, 1 \mathrm{H}, \mathrm{NH}) .{ }^{13} \mathrm{C} \mathrm{NMR}(100$ $\left.\mathrm{MHz}, \mathrm{DMSO}-d_{6}\right) \delta_{\mathrm{C}}(\mathrm{ppm}): 17.90\left(\mathrm{Ar}-\mathrm{OCH}_{2}-\underline{\mathrm{CH}}_{3}\right), 21.06\left(\underline{\mathrm{CH}}_{3}-\mathrm{CH}-\underline{\mathrm{CH}}_{3}\right), 30.23\left(\mathrm{CH}_{3}-\underline{\mathrm{CH}}-\mathrm{CH}_{3}\right)$, $59.00\left(\mathrm{Ar}-\mathrm{OCH}_{2}-\mathrm{CH}_{3}\right), 108.36,110.56,112.36,114.01,116.20,117.01,118.70,120.31,122.00$, 124.30, 126.31, 128.34, 130.21, 132.01, 134.01, 136.07, 138.00, 139.14 (Ar-C) , 163.00 (-CO-NH-). $\mathrm{MS}\left(\mathrm{M}^{+}\right)$: 539.06, Anal. Calcd. for $\mathrm{C}_{24} \mathrm{H}_{22} \mathrm{BrN}_{5} \mathrm{O}_{3} \mathrm{~S}$ (540.43): C 57.51, H 3.07, N 21.34 Found: C 57.45, H 3.22, N 21.20\%. 


\section{(E)-2-(4-chlorobenzylideneamino)-4-ethoxy- $N$-(4-(furan-2-yl)thiazol-2-yl)-6- isopropylpyrimidine-5-carboxamide (5s)}

Yellow solid, yield 79\%, m.p. 173-175 C, IR (KBr, v, $\mathrm{cm}^{-1}$ ): 3365 (N-H Str.), 3053 (Ar C-H Str.), 1698 (-C=O- Str.). ${ }^{1} \mathrm{H}$ NMR (400 MHz, DMSO- $\left.d_{6}\right) \delta_{\mathrm{H}}(\mathrm{ppm}): \delta 1.201-1.250\left(\mathrm{~d}, 6 \mathrm{H},-\mathrm{CH}\left(\mathrm{CH}_{3}\right)_{2}\right)$, 1.290-1.302 (t, 3H, Ar-OCH $\left.2-\mathrm{CH}_{3}\right), 3.202-3.298$ (m, $\left.1 \mathrm{H},-\mathrm{C} \underline{\mathrm{H}}\left(\mathrm{CH}_{3}\right)_{2}\right), 3.900-4.00$ (q, $2 \mathrm{H}, \mathrm{Ar}-$ $\left.\mathrm{OCH}_{2}-\mathrm{CH}_{3}\right), 6.980-7.625(\mathrm{~m}, 8 \mathrm{H}, \mathrm{Ar}-\mathrm{H}), 9.321(\mathrm{~s}, 1 \mathrm{H}, \mathrm{CH}), 10.412(\mathrm{~s}, 1 \mathrm{H}, \mathrm{NH}) .{ }^{13} \mathrm{C} \mathrm{NMR}(100$ $\left.\mathrm{MHz}, \mathrm{DMSO}-d_{6}\right) \delta_{\mathrm{C}}(\mathrm{ppm}): 17.88\left(\mathrm{Ar}-\mathrm{OCH}_{2}-\underline{\mathrm{CH}}_{3}\right), 21.01\left(\underline{\mathrm{CH}}_{3}-\mathrm{CH}-\underline{\mathrm{CH}}_{3}\right), 32.22\left(\mathrm{CH}_{3}-\underline{\mathrm{C}} \mathrm{H}-\mathrm{CH}_{3}\right)$, $58.96\left(\mathrm{Ar}-\mathrm{OCH}_{2}-\mathrm{CH}_{3}\right), 111.01,112.00,114.02,116.53,117.20,118.30,120.45,122.00,124.36$, 126.31, 127.01, 129.30, 131.20, 133.00, 135.01, 137.03, 139.41, 140.21 (Ar-ㅡ), 164.36 (-ㅡ-NH-). $\mathrm{MS}\left(\mathrm{M}^{+}\right)$: 495.11, Anal. Calcd. for $\mathrm{C}_{24} \mathrm{H}_{22} \mathrm{ClN}_{5} \mathrm{O}_{3} \mathrm{~S}$ (495.98): C 58.12, H 4.47, N 14.12 Found: $\mathrm{C}$ 58.18, H 4.20, N 14.06\%.

\section{(E)-2-(3-chlorobenzylideneamino)-4-ethoxy- $N$-(4-(furan-2-yl)thiazol-2-yl)-6- isopropylpyrimidine-5-carboxamide $(5 t)$}

Yellow solid, yield 76\%, m.p. 158-160 C, IR (KBr, v, $\mathrm{cm}^{-1}$ ): 3326 (N-H Str.), 3038 (Ar C-H Str.), 1683 (-C=O- Str.). ${ }^{1} \mathrm{H}$ NMR (400 MHz, DMSO- $\left.d_{6}\right) \delta_{\mathrm{H}}(\mathrm{ppm}): \delta 1.156-1.200\left(\mathrm{~d}, 6 \mathrm{H},-\mathrm{CH}\left(\mathrm{CH}_{3}\right)_{2}\right)$, 1.220-1.300 (t, $\left.3 \mathrm{H}, A r-\mathrm{OCH}_{2}-\mathrm{CH}_{3}\right), 3.220-3.280\left(\mathrm{~m}, 1 \mathrm{H},-\mathrm{C} \underline{\mathrm{H}}\left(\mathrm{CH}_{3}\right)_{2}\right), 3.890-3.941$ (q, $2 \mathrm{H}, \mathrm{Ar}-$ $\left.\mathrm{OCH}_{2}-\mathrm{CH}_{3}\right), 6.980-7.500(\mathrm{~m}, 8 \mathrm{H}, \mathrm{Ar}-\mathrm{H}), 9.365(\mathrm{~s}, 1 \mathrm{H}, \mathrm{CH}), 10.421(\mathrm{~s}, 1 \mathrm{H}, \mathrm{NH}) .{ }^{13} \mathrm{C} \mathrm{NMR}(100$ $\left.\mathrm{MHz}, \mathrm{DMSO}-d_{6}\right) \delta_{\mathrm{C}}(\mathrm{ppm}): 19.03\left(\mathrm{Ar}-\mathrm{OCH}_{2}-\underline{\mathrm{CH}}_{3}\right), 22.78\left(\underline{\mathrm{CH}}_{3}-\mathrm{CH}-\underline{\mathrm{C}} \mathrm{H}_{3}\right), 32.10\left(\mathrm{CH}_{3}-\underline{\mathrm{CH}}-\mathrm{CH}_{3}\right)$, $60.21\left(\mathrm{Ar}-\mathrm{OCH}_{2}-\mathrm{CH}_{3}\right), 110.20,112.03,114.00,115.32,116.36,117.12,119.30,121.01,123.00$, 125.03, 127.41, 129.30, 131.40, 132.01, 135.04, 137.00, 139.06, 140.25 (Ar-C)), 165.00 (-COO-NH-). $\mathrm{MS}\left(\mathrm{M}^{+}\right)$: 495.11, Anal. Calcd. for $\mathrm{C}_{24} \mathrm{H}_{22} \mathrm{ClN}_{5} \mathrm{O}_{3} \mathrm{~S}$ (495.98): $\mathrm{C} 58.12, \mathrm{H}$ 4.47, $\mathrm{N} 14.12$ Found: $\mathrm{C}$ 58.18, H 4.20, N 14.06\%.

\section{CONCLUSION}

Here, we report the synthesis of substituted pyrimidines bearing furanyl thiazole nucleous. It means the method produced a single scaffold with furan, thiazole and pyrimidine hetrocyclic systems.

\section{Acknowledgments}

The authors are thankful to the Head, Department of Chemistry, Saurashtra University, Rajkot for providing ${ }^{1} \mathrm{H}$ NMR, ${ }^{13} \mathrm{C}$ NMR, mass and FT-IR spectroscopy and research facilities.

\section{References}

[1] Lagoja M., et al., Chemistry \& Biodiversity 2 (2005) 1

[2] Agarwal OP., et al., Organic chemistry, Reaction and Reagent (2006) 735

[3] Jain K. S., Chitre T. S., Curr. Sci. 90 (2006) 793

[4] Zhang N., Ayral-Kaloustian S., Nguyen T., Bioorg. Med. Chem. Lett. 17 (2007) 3003

[5] Cocco M. T., Congiu C., Lilliu V., Bioorg. Med. Chem. 14 (2006) 366

[6] Palwinder S., Kamaldeep P., J. Bioorganic and Medicinal Chemistry 14 (2006) 8622

[7] Sirisoma N., Kasibhatla S., Nguyen B., Bioorg. Med. Chem.vol.14 (2006) 7761

[8] Abdel-Gawad S., El-Gaby M., J Chin Chem Soc (2005) 1227

[9] El-Gaby M., Abdel Gawad S., Phosphorus Sulphur Silicon (2006) 279

[10] Selvi S., Nadaraj V., Bioorg Med Chem (2006) 3896 
[11] Gawin R., Clercq E., Naesens L., Bioorg Med Chem 16 (2008) 8379

[12] Gupta P., Srimal R., Avasthi K., Ind. J. Exp Biol. (1995) 38

[13] Camoutsis C., Zoumpoulakis P., Bioorg Med Chem (2008) 1150

[14] Anjaneyulu R., Anjaneyulu K., Couturier E., Biochem Pharmacol (1980) 1879

[15] Samols E., Weir G., Ramseur R., Biochem Pharmacol (1978) 1219

[16] Shayma M., et al., Journal of Al-Nahrain University 14 (2011) 24

[17] Parmar K., Vasava S., Journal of Chemical and Pharmaceutical Research 5 (2013) 222

[18] Mosharef Hossain Bhuiyan., et al., Acta Pharm 56 (2006) 441

[19] Harish kumar., Acta Pharm 58 (2008) 467

[20] Bhalla A., Shamsher S., Canadian Chemical Transactions 3 (2015) 72

[21] Nagah H., Omar Khalid., International Journal of Organic Chemistry 3 (2013) 110

[22] Shahrzad Abdolmohammadi., et al., International Journal of Organic Chemistry 2 (2012) 7

[23] Alya M., Nouria A., Molecules 16 (2011) 5182

[24] Sun L., Wu J., Molecules 16 (2011) 5618

[25] Yun H., Zhao J., Molecules 16 (2011) 10685

[26] Dodson R. M., King L. C., J Am Chem Soc. 67 (1945) 2242.

[27] Khunt H., Pipaliya P., Ghelani S., Naliapara Y. T., International Letters of Chemistry, Physics and Astronomy 24 (2014) 134.

[28] Patel A. S., Khunt H., Babariya J., Ghelani S., Naliapara Y. T., International Letters of Chemistry, Physics and Astronomy 30 (2014) 106.

[29] Gami P., Vilapara K., Khunt H., Babariya J., Naliapara Y. T., International Letters of Chemistry, Physics and Astronomy 30 (2014) 127.

[30] Khunt H., Babariya J., Dedakiya C., Naliapara Y. T., International Letters of Chemistry, Physics and Astronomy 30 (2014) 185.

[31] Khunt H., Babariya J., Naliapara Y. T., International Letters of Chemistry, Physics and Astronomy 31 (2014) 20.

[32] Prajapati D., Vilapara K., Naliapara Y. T., International Letters of Chemistry, Physics and Astronomy 33 (2014) 12.

[33] Kathrotiya H., Gami S., Naliapara Y. T., International Letters of Chemistry, Physics and Astronomy 51 (2015) 125.

[34] Kathrotiya H., Naliapara Y. T., International Letters of Chemistry, Physics and Astronomy 51 (2015) 115.

[35] Kathrotiya H., Naliapara Y. T., International Letters of Chemistry, Physics and Astronomy 52 (2015) 63.

[36] Kathrotiya H., Naliapara Y. T., International Letters of Chemistry, Physics and Astronomy 52 (2015) 74. 